\title{
Research on Image Measuring method based on monocular camera and Ruler
}

\author{
Jun Sun \\ College of Civil Engineering and Architecture, Guizhou University of Engineering \\ Science,Bijie,Guizhou 551700,China
}

\begin{abstract}
Start from the image edge detection, the papers analyze the edge detection principle and some commonly edge detection operator, and on basis of it, we introduced wavelet transform, and proposed an improved multi-scale edge detection algorithm, then new edge thinning algorithm are proposed for the particularity of the edge, finally picking up the features through the mark point target, calculate the final distance results. The results also prove that the proposed scheme is effective, adaptive and accurate, and meets the requirements of real industrial production.
\end{abstract}

KEYWORDS: Digital image; Reference plane; Calibration objects; Wavelet Transformation; Edge Thinning;

\section{INTRODUCTION}

At present, commonly image measuring mainly include the non-contact measuring and contact measuring, the non-contact measurement method is measuring speed fast, and non-contact characteristics, more and more widely used in the practical work. Commonly used non-contact measurement method includes three coordinate measuring machines ranging, tools microscope ranging, laser ranging, level range finder, satellite positioning and based on digital image distance measure. Three coordinate laser range finder and tool microscope is high ranging measurement precision, but the range is limited; laser ranging has higher measurement accuracy, but due to the system of small time interval measurement limitations; level gauge and the latitude and longitude range finder in the measurement of low cost, but the operation is complex, the degree of automation is low; satellite positioning and high degree of automation, but must in measured object is provided with a signal receiving device, and measurement accuracy is limited, in use is rather limited. In recent years, with the development of computer vision technology, digital image measuring distance principle and method become hotspot in the research of non-contact measurement. Based on the digital images ranging must first obtain the internal parameters of the camera, then to determine the external parameters of the camera by the specific standard, establish relations between camera and spatial objects, finally we calculate measured object size according to the principle of projective geometry ${ }^{[1-2]}$.

The gradient operator of the image edge have Roberts, Sobel, Prewitt and Laplace. However, these algorithms cannot guarantee the complete continuous edge extraction, so it can't meet many application occasions in industrial machine vision. The Sobel operator based on the proposed a new algorithm, using the method of measuring the distance in a straight line with calibration and measurement procedure was simple, established mathematical model is a set of linear equations, good convergence, high calculation accuracy, overcomes the defects of the existing fault location methods, it can instead of the current measurement method in a certain extent, and can be widely used in the field of engineering measurement ${ }^{[2]}$. 


\section{Sobel edge detection operator}

\subsection{Basic principle of Sobel operator}

Sobel operator is a common gradient detection operator. Let $\mathrm{f}(\mathrm{x}, \mathrm{y})$ is a digital image function and its first-order differential is based on images of various two-dimensional gradient approximation [3], the gradient of image $\mathrm{f}(\mathrm{x}, \mathrm{y})$ the can be defined as a follows vector at a point $(\mathrm{x}, \mathrm{y})$ :

$$
\nabla f(x, y)=\left[\begin{array}{l}
G_{x} \\
G_{y}
\end{array}\right]=\left[\begin{array}{l}
\frac{\partial f}{\partial x} \\
\frac{\partial f}{\partial y}
\end{array}\right]
$$

In the formula $(1), f(x, y)$ is the gradient of the image, including the change information of gray, and its value can be calculated by formula (2).

$$
\nabla f(x, y)=\left[\left(\frac{\partial f}{\partial x}\right)^{2}+\left(\frac{\partial f}{\partial y}\right)^{2}\right]^{\frac{1}{2}}
$$

For the convenience of calculation, we usually use formula (3) to calculate the gradient image in the actual edge detection. the GX and Gy in Type (3) is convolution formula, were used to calculated the horizontal direction and the vertical direction partial differential, Fig.1 (left) is pixel (x,y) and its neighborhood pixels, Fig.1 (right) is convolution template (Sobel operator), partial differential formula is as shown in formula (4) and (5).

$$
\begin{gathered}
f(x, y)=\max \left\{\left|G_{x}\right|,\left|G_{y}\right|\right\} \\
G_{x}=\{f(x-1, y+1)+2 f(x, y+1)+f(x+1, y+1)\}-\{f(x-1, y-1)+2 f(x, y-1)+f(x+1, y-1)\} \\
G_{y}=\{f(x+1, y-1)+2 f(x+1, y)+f(x+1, y+1)\}-\{f(x-1, y-1)+2 f(x-1, y)+f(x-1, y+1)\}
\end{gathered}
$$

The horizontal gradient direction template in Figure 2 is used to detect the vertical edge, and the vertical gradient direction template is used to detect the horizontal edge of the image.

\begin{tabular}{|c|c|c|}
\hline$(x-1, y-1)$ & $(x, y-1)$ & $(x+1, y-1)$ \\
\hline$(x-1, y)$ & $(x, y)$ & $(x+1, y)$ \\
\hline$(x-1, y+1)$ & $(x, y+1)$ & $(x+1, y+1)$ \\
\hline
\end{tabular}

\begin{tabular}{|l|l|l|}
\hline-1 & 0 & 1 \\
\hline-2 & 0 & 2 \\
\hline-1 & 0 & 1 \\
\hline
\end{tabular}

\begin{tabular}{|c|c|c|}
\hline 1 & 2 & 1 \\
\hline 0 & 0 & 0 \\
\hline-1 & -2 & -1 \\
\hline
\end{tabular}

Fig.1 (left) A pixel and its neighborhood pixels Fig.1(right) Sobel detection operator

Since the gray level changes near the edge of the image are relatively large, the pixels can be used as the edge points of the gradient values in the neighborhood of a certain threshold. Visible, Sobel algorithm is simple, fast detection speed, but it is only the two direction template, the positioning accuracy is not high, on the horizontal and vertical edge gradient change sensitive and of complex texture image (there are many cable to edge detection effect is not very ideal, and the classical Sobel operator will gradient value is greater than or equal to the set point of the threshold as the output of the edge point, this will cause the misjudgment of edge points, because some noise gradient value is great. In addition, the edge of the Sobel operator is not refined, and the image edge is rough.

\subsection{Edge detection template of Sobel operator based on eight direction}

This algorithm in the paper uses the size of eight $5 \times 5$ direction template. The 8 directions are $0,22.5,45,67.5090,112.5,135$ and 157.5 degrees, as shown in figure 2 . 


\begin{tabular}{|c|c|c|c|c|c|c|c|c|c|c|c|c|c|c|c|c|c|c|c|}
\hline 0 & 0 & 0 & 0 & 0 & 0 & 0 & 0 & 0 & 0 & 0 & -1 & 0 & 1 & 0 & 0 & 0 & 1 & 0 & 0 \\
\hline-1 & -2 & -4 & -2 & -1 & 0 & -2 & -4 & -2 & 0 & 0 & -2 & 0 & 2 & 0 & 0 & -2 & 4 & 2 & 0 \\
\hline 0 & 0 & 0 & 0 & 0 & -1 & -4 & 0 & 4 & 1 & 0 & -4 & 0 & 4 & 0 & 0 & -4 & 0 & 4 & 0 \\
\hline 1 & 2 & 4 & 2 & 1 & 0 & 2 & 4 & 2 & 0 & 0 & -2 & 0 & 2 & 0 & 0 & -2 & -4 & 2 & 0 \\
\hline 0 & 0 & 0 & 0 & 0 & 0 & 0 & 0 & 0 & 0 & 0 & -1 & 0 & 1 & 0 & 0 & 0 & -1 & 0 & 0 \\
\hline \multicolumn{5}{|c|}{$0^{0}$} & \multicolumn{5}{|c|}{$22.5^{0}$} & \multicolumn{5}{|c|}{$90^{\circ}$} & \multicolumn{5}{|c|}{$112.5^{6}$} \\
\hline 0 & 0 & 0 & -1 & 0 & 0 & 0 & -1 & 0 & 0 & 0 & 1 & 0 & 0 & 0 & 0 & 0 & 0 & 0 & 0 \\
\hline 0 & -2 & -4 & 0 & 1 & 0 & -2 & -4 & 2 & 0 & -1 & 0 & 4 & 2 & 0 & 0 & 2 & 4 & 2 & 0 \\
\hline 0 & -4 & 0 & 4 & 0 & 0 & -4 & 0 & 4 & 0 & 0 & -4 & 0 & 4 & 0 & -1 & -4 & 0 & 4 & 1 \\
\hline-1 & 0 & 4 & 2 & 0 & 0 & -2 & 4 & 2 & 0 & 0 & -2 & -4 & 0 & 1 & 0 & -2 & -4 & -2 & 0 \\
\hline 0 & 1 & 0 & 0 & 0 & 0 & 0 & 1 & 0 & 0 & 0 & 0 & 0 & -1 & 0 & 0 & 0 & 0 & 0 & 0 \\
\hline
\end{tabular}

Fig.2 the algorithm of convolution template in this paper

The template is as shows in Figure 2, the weights of different position $\omega(m, n)$ is calculated under the given type (4-6).

$$
\begin{aligned}
& d(m, n)=\sqrt{(i-m)^{2}+(j-n)^{2}} \\
& \ln g(m, n)=-\left[d(m, n)^{2}-u\right] \ln 2 \\
& w(m, n)=\sqrt{(i-m)^{2}+(j-n)^{2}}
\end{aligned}
$$

$\mathrm{d}(\mathrm{m}, \mathrm{n})$ are the Euclidean distance between the template center(i,j) and the template elements of coordinates $(m, n), u$ is the adjustment coefficient (about, and the template size is $u=3), g(m, n)$ is real weight at $(m, n)$. To simplify the calculation, the integer $g(m, n)$ is as the template elements. "[]"in type (6) is on behalf of integer arithmetic.

Figure 3 depicts the distance and weight relation between template pixels to the center pixel based on the type (5). The abscissa is shown in the pixel to pixel template center distance, the vertical axis is the corresponding weight. We can see from Figure 3, the center pixel template pixel to the smaller the distance, the greater weight; otherwise, the template pixels to the center pixel distance is, the smaller the weight.

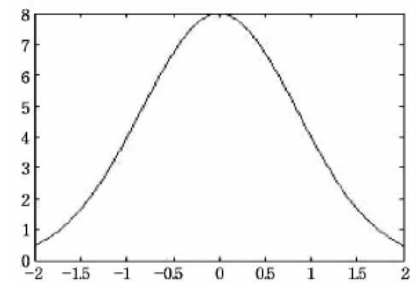

Fig.3 Schematic diagram of weight and distance
The algorithm are using eight direction template and the corresponding Image data to do convolution. Algorithm and gray 8 cache larger value is as the current pixel output value.

\section{3 algorithm implementation}

Suppose matrix $[f(i, j)]$ said image to be detected (The $(i, j)$ represent the coordinates of each pixel), $f(i, j)$ represent the gray value of image in $(i, j)$ of, $\omega[f(i, j)]$ said the $5 \times 5$ window taking the point $(i, j)$ as the center, $g(i, j)$ is point $(i, j)$ gray output after the image edge detection.

Algorithm steps:

Suppose $\operatorname{mk}(\mathrm{k}=1,2, \ldots, 8)$ is $5 \mathrm{X} 5$ templates are given in Fig. 2, $\mathrm{n}$ is Integer array with 8 elements. For each pixel in the image (coordinate $(\mathrm{I}, \mathrm{J})$ ) were treated as follows:

Step 1:for processing pixel $(i, j)$, using template $\operatorname{mk}(\mathrm{k}=1,2, \ldots, 8)$ and $\mathrm{w}[\mathrm{f}(\mathrm{i}, \mathrm{j})]$ to be convolution, and the absolute value of the convolution result stored in an array element $\mathrm{n}[\mathrm{k}](\mathrm{k}=1,2, \cdots, 8)$;

Step 2: find maximum value in all elements in array obtained by step 1 .

$\mathrm{k}=1$;

2. 1 initialize $\max =\mathrm{n}[0]$, $\max =\mathrm{n}[\mathrm{k}]$

$2, \quad 2$ if $\mathrm{n}[\mathrm{k}]>\max$, so set

2. 3 if $\mathrm{k}<8$, so $\mathrm{k}=\mathrm{k}+1$, jump to 2.2; Otherwise to Step 3;

Step 3: Take max to $g(i, j)$, which is as the output of the pixel value. 


\section{Experiment and measure analysis}

\subsection{Calculation of measure results}

As is shown in figure 4, we can calculated the final results by the following formula after mark target point:

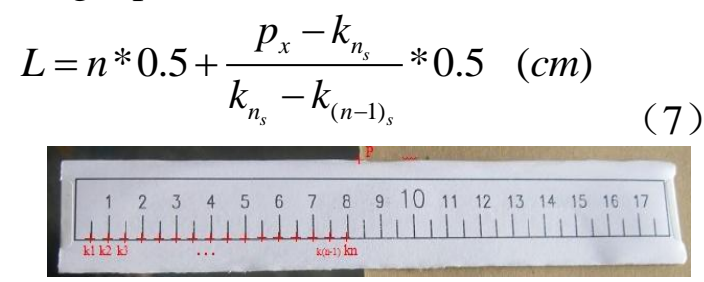

Fig.4 Marked sample graph

$\mathrm{P}$ is intersection between the scale and the edge of the paper, $n$ is scale number of P-point mark on the left, $\mathrm{PX}$ is a P-point abscissa, kn, $\mathrm{K}$ ( $\mathrm{n}-1$ is the abscissa of $\mathrm{N}, \mathrm{N}-1$ points, ruler of the precision is $0.5 \mathrm{~cm}$. The distance $\mathrm{L}$ in the formula (7) is divided into two parts: the first part is determined by the number of points and the accuracy of the ruler directly, and the second part is estimated by the number of pixels.

\subsection{The effect diagram of refinement algorithm processing}

Figure 5 (a) is the first thinning algorithm based on improved edge center extraction algorithm treatment effect diagram, from Figure 5 can be seen that it keep the edge of the original topology after the thinning algorithm edges comply with the thinning algorithm, center and single pixel wide requirements, although the perpendicular at the edge of the edge line is not extracted, but the characteristics did not affect subsequent processing instead to the filling of vacancies and feature extraction brings convenience. Figure 5 (b) is an improved refinement algorithm of second vacancies fill algorithm processing effect, the algorithm is good to fill the edge of the extraction algorithm cannot extract the edge of the line. Figure 5 (d) is the result of the final tagging and measure results.

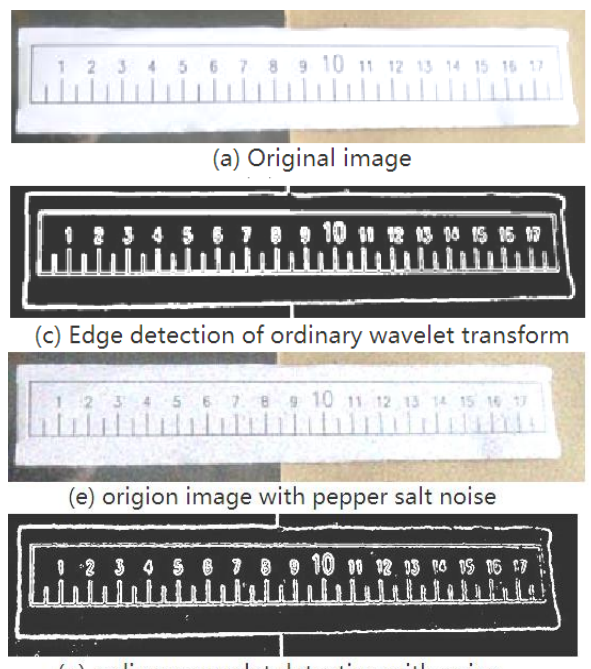

(g) ordinary wavelet detection with noise
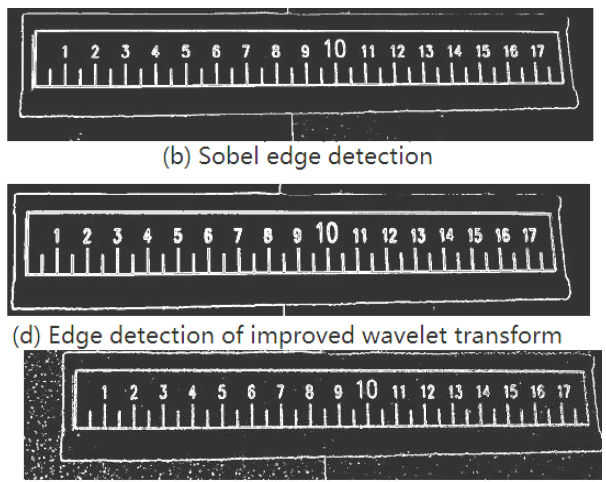

(f) Sobel edge detection with noise

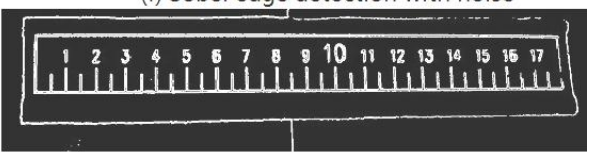

(h) improved wavelet edge detection with noise

Fig.5 Edge detection effect diagram 

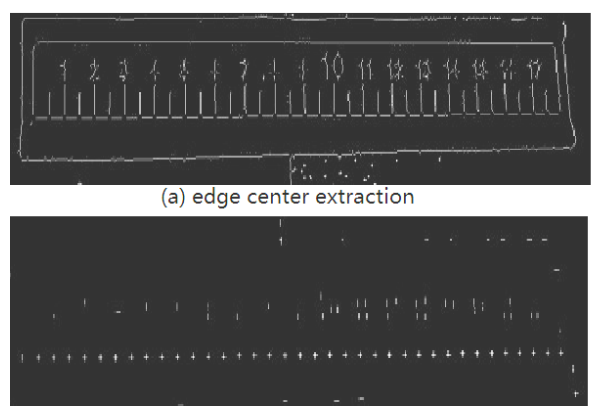

(c) new image after fill vacancies

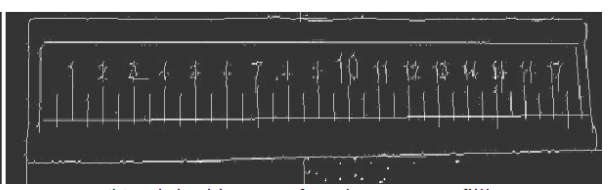

(b) original image after the vacancy filling

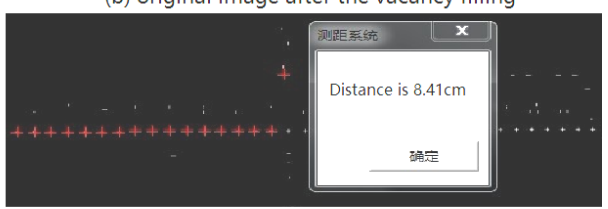

(d) reading

Fig.6 Refinement and reading effect diagram

\subsection{Ranging results comparison}

Table 1 is the outcome of each experiment, the third row in the table is using Sobel operator to be edge detection, and the fourth row is the ordinary wavelet transform edge detection. At the end of the row is the improved algorithm of edge detection. From the results of the previous three results, there are three methods that can detect the image ranging from the ordinary noise. The results can be compared with the results of the method. When the image is noisy, the accuracy of the results obtained by the edge detection using the Sobel operator is very high. However, when the image is added to a certain noise, the results of the ordinary Sobel operator have a lot of error.

Table.1 Results of the experimental measuring

\begin{tabular}{c|c|c|c|c|c|c|c|c|c|c|c}
\hline Method & 1 & 2 & 3 & 4 (noise) & 5 (noise) & Method & 1 & 2 & 3 & 4 (noise) & 5 (noise) \\
\hline $\begin{array}{c}\text { Actual distance } \\
(\mathrm{cm})\end{array}$ & 6.04 & 8.40 & 14.00 & 8.40 & 14.00 & $\begin{array}{c}\text { (wavelet) } \\
\text { measurement }\end{array}$ & 6.31 & 8.31 & 14.08 & 8.33 & 14.08 \\
\hline $\begin{array}{c}\text { (Sobel) } \\
\text { Measurement }\end{array}$ & 6.37 & 8.37 & 14.05 & 7.93 & 13.01 & $\begin{array}{c}\text { (improve) } \\
\text { measurement }\end{array}$ & 6.36 & 8.36 & 14.02 & 8.37 & 14.03 \\
\hline
\end{tabular}

\section{Conclusions}

Table 1 is the outcome of each experiment. From the results of the previous three results, there are three methods that can detect the image ranging from the ordinary noise. The results can be compared with the results of the method. When image have no noise, using Sobel operator for edge detection test result accuracy is very high, but when the image and the noise, ordinary Sobel test results has a lot of error. Actually, after adding noise, Sobel operator edge detection result generate many false edge, which lead edge thinning the edge tracking failure, eventually making part of the target point cannot be located, it can be seen from equation (4), if less calibration of a target point and the gap will is $0.5 \mathrm{~cm}$ precision ruler, so the table in bold in the two experimental results in errors. The edge detection using wavelet transform is less affected by the noise, and the ranging results are relatively stable. Compared with the last two rows of data, the improved wavelet transform edge detection algorithm is more than the ordinary wavelet edge detection algorithm.

\section{Acknowledgement}

Bijie university Joint Fund Project(Guizhou science joint J LKB[2012]08)

\section{Reference}

[1] Su Chengzhi, Wang Enguo, Hao Jiangtao, Cao Guohua, Xu Hongji. Image distortion correction in the measurement of plane geometry $[\mathrm{J}]$. Optics and precision engineering, 2011, 19 (1):161-166.

[2] Li Lidong, Liu Jiaomin. Research on a 
single view camera calibration and plane measurement $[\mathrm{J}]$. Journal of Hebei University of Science and Technology, 2005, 26 (1):47-50.

[3] Gao Junchai, Lei Zhiyong, Wang Zemin. Camera calibration for high precision measurement [J]. Electro-optic and control, 2011, 18 (2):93-96.

[4] Wang Fuzhi, Yang Ping, Huang Dagui. The two-dimensional image measurement system based on steady camera calibration and sub-pixel algorithm [J]. Computer measurement and control, 2005, 13 (12):1325-1328.
[5] Wang Lan. Research on Algorithm of image edge detection based on Wavelet Transform [D]. Wuhan: Wuhan University of Science and Technology.2011

[6] Li Bicheng, Peng Tianqiang, Peng Bo. Intelligent image processing technology [M]. Beijing: Publishing House of electronics industry, 2004

[7] Wang Weizhao, Li Zhanxian, Zhang Jin. Image multiscale edge detection algorithm based on Wavelet Transform [J]. Journal of Hebei Polytechnic University (NATURAL SCIENCE EDITION), 2009, 8:88-93. 